\title{
Production rate of hard thermal photons and screening of quark mass singularity ${ }^{\star}$
}

\author{
R. Baier ${ }^{1}$, H. Nakkagawa ${ }^{1,2}$, A. Niégawa ${ }^{1,3}$, K. Redlich ${ }^{1,4}$ \\ 1 Fakultät für Physik, Universität Bielefeld, W-4800 Bielefeld 1, Federal Republic of Germany \\ 2 Institute for Natural Science, Nara University, Nara 631, Japan \\ ${ }^{3}$ Department of Physics, Osaka City University, Osaka 558, Japan \\ ${ }^{4}$ Department of Theoretical Physics, University of Wroclaw, PL-50200 Wroclaw, Poland
}

Received 26 July 1991

\begin{abstract}
The emission of hard real photons from a quark-gluon plasma at high temperatures is studied. At lowest order the QCD processes for quark-antiquark annihilation $(q \bar{q} \rightarrow \gamma g)$ and for Compton scattering $(q g \rightarrow \gamma q)$ lead to logarithmically divergent thermal production rates for massless quarks. By applying recently developed resummation methods of finite temperature perturbation theory we show that effects due to Landau damping on the exchanged quark provide finite rates. Comparisons with previous estimates and phenomenological implications are discussed.
\end{abstract}

\section{Introduction}

Ultrarelativistic heavy-ion experiments at CERN [1], and in the future at Brookhaven RHIC, focus on energetic direct photons as promising signatures of the expected quark-gluon plasma phase. Already many investigations [2-8] are devoted to this signal, however, it seems still necessary and possible to improve on the theoretical predictions.

In this paper we concentrate on real photons emitted from a quark-gluon plasma at high temperatures. For massless quarks the basic QCD processes, quark-antiquark annihilation $(q \bar{q} \rightarrow \gamma g)$ and Compton scattering $(q g \rightarrow \gamma q)$, lead to (logarithmically) divergent production rates at leading order of the perturbative expansion. This is in contrast to thermal dilepton pair, i.e. heavy photon production, where the quark mass singularities cancel -at least to two-loop order $[9,10]$.

For real photons these mass singularities therefore have to be shielded by thermal effects in order to present infrared safe calculations, as it was already conjectured

* Supported in part by the Minister for Research and Technology (BMFT) of the Federal Republic of Germany under contract 06BI-701 and partially shown by Kajantie and Ruuskanen [2] some time ago.

Recently Pisarski $[11,12]$, together with Braaten [13], and Frenkel and Taylor [14] proposed and developed a resummation method for finite temperature perturbation theory [15], which allows to investigate screening effects in a systematic way. Examples of processes, in which massless gauge bosons, gluons [16] and photons [17], are exchanged, could be successfully treated, showing that dynamical screening indeed cures logarithmic mass divergencies.

In case of real photons massless quark exchange is present. It is expected that screening should work here as well as already supposed by Baym et al. [16]. In the following we quantitatively apply the resummed perturbative expansion showing explicitly in the real-time framework that effects due to Landau damping on the exchanged quark provide indeed finite rates for hard photons [18].

\section{Thermal photon rates}

\subsection{Soft contribution}

According to the discussions and results given by Weldon [19] and by Kobes and Semenoff [20] rates at finite temperature may be calculated from the absorptive parts of thermal Green's functions [21]. Here we are interested in the emission rate of real photons from a thermalized plasma of quarks and gluons. The corresponding relation between the differential rate and the on-shell photon polarization tensor reads

$E \frac{\mathrm{d} W^{\gamma}}{\mathrm{d}^{3} k}=\frac{1}{(2 \pi)^{3}} n_{B}(E) \operatorname{Im} \Pi_{\mu}^{\mu}(E, \mathbf{k})$,

after summing the polarizations of the photon. The photon energy is denoted by $E$, and its momentum by $\mathbf{k}, E=|\mathbf{k}|$. The presence of the Bose-Einstein factor

$n_{B}(E)=1 /[\exp (E / T)-1]$ 


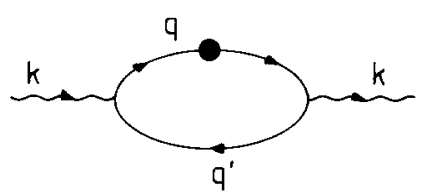

Fig. 1. Effective one-loop diagram for the production of a real photon (weavy line) with momentum $k$. The effective quark propagator is indicated by a blob

reflects thermal equilibrium and detailed balance between the production (emission) and decay (absorption) rates.

When applying the resummed perturbative expansion it is helpful to distinguish two energy scales [11-13]: one of the order of the temperature $T$ and one of $g T$ with $g$ the strong coupling constant. The external photon is assumed to be hard, its energy should be even larger than the temperature of the heat bath. In the high temperature limit the extensive studies $[11-14,17]$ require to consider the effective one-loop diagram of Fig. 1 with a quark loop with one soft (dressed) and one hard (bare) quark line (a diagram interchanging the blob in Fig. 1 has to be added). No effective vertices have to be included.

In order to evaluate the rate (1), we continue in the real-time framework $[15,22]$. With the generalized Cutkosky rules at finite temperature $[20,21]$ the effective one-loop expression for the discontinuity of $\Pi_{\mu}^{\mu}$ can be written as

$$
\begin{aligned}
\operatorname{Im} \Pi_{\mu}^{\mu}(E, \mathbf{k})= & \frac{1}{2}[\exp (E / T)-1] e^{2} e_{q}^{2} N_{c} \\
& \cdot \int \frac{\mathrm{d}^{4} q}{(2 \pi)^{4}} \operatorname{tr}\left[\gamma^{\mu} \mathrm{i}^{*} S^{-}(q) \gamma_{\mu} \mathrm{i} S^{+}\left(q^{\prime}\right)\right. \\
& \left.+\gamma^{\mu} \mathrm{i} S^{-}(q) \gamma_{\mu} \mathrm{i}^{*} S^{+}\left(q^{\prime}\right)\right],
\end{aligned}
$$

with $q^{\prime}=q-k$ (cf. Fig. 1). It holds for one quark flavour with charge $e_{q}$, and for $N_{c}$ colour degrees of freedom.

The bare thermal propagator for massless fermions is given by

i $S^{ \pm}(q)=2 \pi \phi\left[\theta\left( \pm q^{0}\right)-n_{F}\left(q^{0}\right)\right] \delta\left(q^{2}\right)$,

where the Fermi-Dirac factor is

$n_{F}\left(q^{0}\right)=1 /\left[\exp \left(\left|q^{0}\right| / T\right)+1\right]$.

The complete fermion propagator is expressed in terms of the complex self-energy function $\Sigma$ by

$\mathrm{i}^{*} S^{ \pm}(q)=2\left[\theta\left( \pm q^{0}\right)-n_{F}\left(q^{0}\right)\right] \operatorname{Re}\left(\frac{\mathrm{i}}{q-\Sigma(q)+\mathrm{i} \varepsilon}\right)$.

Klimov [23] and Weldon [24] have first calculated the thermal one-loop quark self-energy function. Its properties are also extensively discussed in $[13,25]$. The leading term in $T$, which is gauge invariant, dominating at soft momentum $q$ and originating from the hard thermal loop is

$$
\begin{aligned}
\operatorname{Re}\left(\frac{\mathrm{i}}{q-\Sigma\left(q^{0}, q\right)+\mathrm{i} \varepsilon}\right)= & \frac{1}{2}\left(\gamma^{0}-\gamma \cdot \hat{\mathbf{q}}\right) \operatorname{Im} \frac{1}{D_{+}\left(q^{0}, q\right)} \\
& +\frac{1}{2}\left(\gamma^{0}+\gamma \cdot \hat{\mathbf{q}}\right) \operatorname{Im} \frac{1}{D_{-}\left(q^{0}, q\right)},
\end{aligned}
$$

where

$\hat{\mathbf{q}}=\mathbf{q} / q, \quad q=|\mathbf{q}|$.

The fermionic excitations contain modes with positive as well as negative helicity/chirality ratio. They are determined by the two functions

$D_{ \pm}\left(q^{0}, q\right)=-q^{0} \pm q+\frac{m_{f}^{2}}{2 q}\left[\left(1 \mp \frac{q^{0}}{q}\right) \ln \frac{q^{0}+q}{q^{0}-q} \pm 2\right]$.

The fermion mass induced by temperature is for QCD given by

$$
\begin{aligned}
m_{f}^{2} & =\frac{g^{2}}{2 \pi^{2}} C_{F} \int_{0}^{\infty} E \mathrm{~d} E\left(n_{B}(E)+n_{F}(E)\right) \\
& =g^{2} C_{F} T^{2} / 8=\frac{2 \pi}{3} \alpha_{\mathrm{s}} T^{2}
\end{aligned}
$$

in terms of the strong coupling constant $\alpha_{\mathrm{s}}$. Below the light-cone-the important region for the processes with quark exchange-the functions $D_{ \pm}$possess a non-zero imaginary part due to the Landau damping mechanism, as discussed in [11-13]. In the real-time framework it is obtained with the help of Feynman's prescription [15], here in the logarithmic function by $q^{0} \rightarrow q^{0}+\mathrm{i} \varepsilon q^{0}$ :

$\ln \frac{q^{0}+q}{q^{0}-q}=\ln \left|\frac{q^{0}+q}{q^{0}-q}\right|-\mathrm{i} \pi \varepsilon\left(q^{0}\right) \theta\left(q^{2}-q^{0^{2}}\right)$,

or equivalently by the direct one-loop calculation of $\operatorname{Im} \Sigma$ using the cutting rules as done in [20] (for the massive case).

Inserting these fermionic propagators into (3) allows to evaluate the rate (1). As already discussed in [17] it is reasonable to introduce an arbitrary momentum scale $q^{*}$ of order $\sqrt{g} T$, and to restrict the momentum transfer to the soft region $q<q^{*}$ : this allows to simplify the kinematics e.g. by $q_{\mu}^{\prime} \simeq-k_{u}$ and $q^{\prime 2} \simeq-2 k \cdot q$, when the hard momentum flow follows the bare quark line (Fig. 1). Keeping in mind that $E>T>q^{*}>m_{f}$ the soft contribution is evaluated as

$$
\begin{aligned}
\left.E \frac{\mathrm{d} W^{\gamma}}{\mathrm{d}^{3} k}\right|_{\text {soft }} \simeq & \frac{e_{q}^{2} \alpha}{4 \pi^{4}} N_{c} \mathrm{e}^{-E / T} \int_{0}^{q^{*}} q \mathrm{~d} q \\
& \cdot \int_{-q}^{+q} \mathrm{~d} q^{0} \varepsilon\left(q^{0}\right)\left[\left(1-\frac{q^{0}}{q}\right) \operatorname{Im} \frac{1}{D_{+}\left(q^{0}, q\right)}\right. \\
& \left.+\left(1+\frac{q^{0}}{q}\right) \operatorname{Im} \frac{1}{D_{-}\left(q^{0}, q\right)}\right] .
\end{aligned}
$$

That the exchanged quark propagation is screened may already be seen when the properties of the functions $D_{ \pm}$for small space-like momenta are investigated. Especially, in the static limit and $q \rightarrow 0$

$\operatorname{Im} \frac{1}{D_{ \pm}} \approx \frac{q}{q^{2}+m_{f}^{2}\left(4+\pi^{2}\right) / 8}$

which produces in the integral of (11) a logarithmic behaviour proportional to $\ln \left(q^{*} / m_{f}\right)$. In more detail using the property

$\operatorname{Im} D_{ \pm}\left(-q^{0}, q\right)=-\operatorname{Im} D_{\mp}\left(q^{0}, q\right)$, 
and the sum rule, which follows from the properties of the functions $D_{ \pm}$of eq. (8),

$$
\int_{-\infty}^{+\infty} \mathrm{d} q^{0} \varepsilon\left(q^{0}\right)\left(1-\frac{q^{0}}{q}\right) \operatorname{Im} \frac{1}{D_{+}\left(q^{0}, q\right)}=0
$$

the $q^{0}$ integration in (11) is easily performed, since above the light-cone quasi-particle modes are present:

$$
\operatorname{Im} \frac{1}{D_{ \pm}(\omega, q)}=\frac{\pi}{2 m_{f}^{2}}\left(\omega_{ \pm}^{2}-q^{2}\right) \delta\left(\omega-\omega_{ \pm}(q)\right), \quad \omega>q .
$$

The frequencies $\omega_{ \pm}(q)$ are determined by the zeros of $D_{ \pm}\left(\omega_{ \pm}, q\right)=0$. The soft contribution then becomes

$$
\begin{aligned}
\left.E \frac{\mathrm{d} W^{\gamma}}{\mathrm{d}^{3} k}\right|_{\text {soft }}= & \frac{e_{q}^{2} \alpha}{4 \pi^{3}} N_{c} \mathrm{e}^{-E / T} \cdot \frac{1}{m_{f}^{2}} \int_{0}^{q^{*}} \mathrm{~d} q\left[\left(\omega_{+}-q\right)\left(\omega_{+}^{2}-q^{2}\right)\right. \\
& \left.-\left(\omega_{-}+q\right)\left(\omega_{-}^{2}-q^{2}\right)\right] .
\end{aligned}
$$

A further simplification is obtained with the identities

$\left(\omega_{ \pm} \mp q\right)\left(\omega_{ \pm}^{2}-q^{2}\right) / m_{f}^{2}=\omega_{ \pm}-q \frac{\mathrm{d} \omega_{ \pm}}{\mathrm{d} q}$.

Recalling the properties of the dispersion poles $\omega_{ \pm}(q)$ at small and large momenta [23-25]:

$\left(\omega_{+}-\omega_{-}\right) \underset{q \rightarrow 0}{\longrightarrow} \frac{2 q}{3}+\ldots$,

$\left(\omega_{+}-\omega_{-}\right) \underset{q \rightarrow \infty}{\longrightarrow} \frac{m_{f}^{2}}{q}+\ldots$,

the logarithmic dependence on $q^{*}$ is extracted in a straightforward way. The final expression may be cast into the form

$$
\begin{aligned}
\left.E \frac{\mathrm{d} W^{\gamma}}{\mathrm{d}^{3} k}\right|_{\text {soft }} & \frac{e_{q}^{2} \alpha \alpha_{\mathrm{s}}}{2 \pi^{2}} T^{2} \mathrm{e}^{-E / T}\left[\ln \left(\frac{q^{*}}{m_{f}}\right)^{2}-1\right. \\
& \left.+\int_{0}^{\infty} \mathrm{d} q\left(2 \frac{\omega_{+}-\omega_{-}}{m_{f}^{2}}-\frac{2}{q+m_{f}}\right)\right],
\end{aligned}
$$

where terms of $\mathcal{O}\left(q^{*} / T\right)$ are neglected. The remaining integral in which we put $q^{*} \rightarrow \infty$ is computed numerically with the value of -0.31 .

We successfully confirmed this result of (17) by applying the imaginary-time methods followed by the appropriate analytic continuation as described in $[17,26]$.

\subsection{Hard contribution}

Now, we need the hard part of the real photon rate, which can be obtained from first-order perturbation theory. Two basic processes contribute, the quark-antiquark annihilation process

$q\left(p_{1}\right)+\bar{q}\left(p_{2}\right) \rightarrow \gamma(k)+g\left(k^{\prime}\right)$,

and the Compton process

$q\left(p_{1}\right)+g\left(p_{2}\right) \rightarrow \gamma(k)+q\left(k^{\prime}\right)$.

The Feynman diagrams for the amplitudes of these reactions are shown in Fig. 2.

We organize the calculation in the following way: first we determine the rate which results from these processes
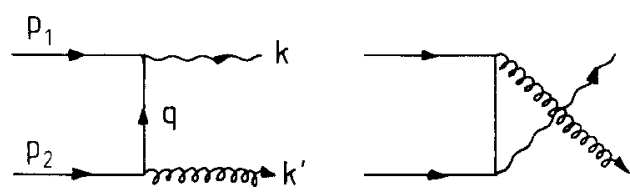

(a)
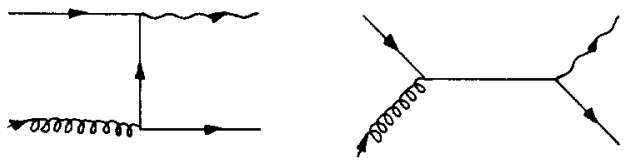

(b)

Fig. 2 a,b. a Annihilation $(q \bar{q} \rightarrow \gamma g)$ and b Compton scattering $(q g \rightarrow \gamma q)$ graphs for real photon production. Weavy (curly) line denotes the photon (gluon)

assumed to be valid over the whole phase-space. Next we subtract the soft part with the cut $q<q^{*}$ introduced before.

The differential rate due to annihilation, (18), is defined by

$$
\begin{aligned}
E \frac{\mathrm{d} W^{\mathrm{ann}}}{\mathrm{d}^{3} k}= & \frac{e_{q}^{2} \alpha \alpha_{\mathrm{s}}}{\pi^{6}} \int \frac{\mathrm{d}^{3} p_{1}}{2 E_{1}} n_{F}\left(E_{1}\right) \int \frac{\mathrm{d}^{3} p_{2}}{2 E_{2}} n_{F}\left(E_{2}\right) \\
& \cdot \int \frac{\mathrm{d}^{3} k^{\prime}}{2 E^{\prime}}\left(1+n_{B}\left(E^{\prime}\right)\right) \delta^{4}\left(p_{1}+p_{2}-k^{\prime}-k\right) \\
& \cdot\left[\frac{u}{t-m^{2}}+\frac{t}{u-m^{2}}\right],
\end{aligned}
$$

with the Mandelstam variables,

$s=\left(p_{1}+p_{2}\right)^{2}, \quad t=\left(p_{1}-k\right)^{2}, \quad s+t+u=0$.

The $m$ dependence in the propagators is introduced as an infrared cut-off in order to keep the expressions finite in the intermediate steps of the calculation. Finally the limit $m \rightarrow 0$ is taken. This procedure allows to compare with results published in the literature, e.g. in $[5,7]$. The integral of (20) is worked out by first transforming to $s-$ and $t-$ integrations as described in [6]. Then the interesting limit of large photon energy $E>T$ is explored for which in leading order the rate becomes

$$
\begin{aligned}
E \frac{\mathrm{d} W^{\mathrm{ann}}}{\mathrm{d}^{3} k} \simeq & \frac{e_{q}^{2} \alpha \alpha_{\mathrm{s}}}{4 \pi^{2}} T^{2} \mathrm{e}^{-E / T} \\
& \cdot\left[\ln \frac{4 T^{2}}{m^{2}}+\frac{4}{3} \ln \frac{E}{T}-\frac{4}{3}-\frac{2}{3} \gamma+\frac{4}{3} \frac{\zeta^{\prime}(2)}{\zeta(2)}\right. \\
& \left.+\frac{8}{\pi^{2}} \sum_{k, l=0}^{\infty}(-)^{k} \frac{\ln (k+1)(l+1)}{(k+l+2)^{2}}\right] .
\end{aligned}
$$

$\gamma$ is Euler's constant, and $\zeta$ is the Riemann zeta function: $\frac{\zeta^{\prime}(2)}{\zeta(2)} \simeq-0.57$.

The double-sum, which has its origin in the distributions $n_{F}$ and $n_{B}$ in the initial and final state, respectively, is 
evaluated numerically,

$$
\sum_{k, l=0}^{\infty}(-)^{k} \frac{\ln (k+1)(l+1)}{(k+l+2)^{2}} \simeq 0.26 \text {. }
$$

The singular logarithmic mass behaviour is present in (21), clearly showing that massless quark exchange leads to an infinite rate at leading order of the perturbative expansion.

In an analogous way the contribution from Compton scattering, (19), is treated. When added to the annihilation part the sum is

$$
\begin{aligned}
E \frac{\mathrm{d} W^{\mathrm{ann}+\text { Compton }}}{\mathrm{d}^{3} k} \simeq & \frac{e_{q}^{2} \alpha \alpha_{\mathrm{s}}}{2 \pi^{2}} T^{2} \mathrm{e}^{-E / T} \\
& \cdot\left[\ln \frac{4 E T}{m^{2}}-\frac{1}{2}+\frac{\ln 2}{3}-\gamma+\frac{\zeta^{\prime}(2)}{\zeta(2)}\right] .
\end{aligned}
$$

The calculation is then repeated, but with the constraint $q<q^{*}\left(q^{*}>m\right)$, and including the kinematical approximations already mentioned in connection with (11). An independent way is to start from (3) and expanding (6), such that only the term proportional to

$$
\frac{\mathrm{i}}{\phi}(-\mathrm{i} \Sigma(q)) \frac{\mathrm{i}}{\dot{q}}
$$

is kept. In this case the boundaries of the $\left(q^{0}, q\right)$ integrations (cf. (11)) have to be carefully chosen: $m \leq q \leq q^{*}$, $-\sqrt{q^{2}-m^{2}} \leq q^{0} \leq \sqrt{q^{2}-m^{2}}$, before the limit $m \rightarrow 0$ is performed. These two ways give the same result, which is then subtracted from (23). Thus we obtain the hard contribution in the kinematical regime $q>q^{*}$ due to hard quark exchange in the processes of (18) and (19),

$$
\begin{aligned}
\left.E \frac{\mathrm{d} W^{\gamma}}{\mathrm{d}^{3} k}\right|_{\text {hard }} \simeq & \frac{e_{q}^{2} \alpha \alpha_{s}}{2 \pi^{2}} T^{2} \mathrm{e}^{-E / T} \\
& \cdot\left[\ln \frac{E T}{q^{* 2}}+\frac{3}{2}+\frac{\ln 2}{3}-\gamma+\frac{\zeta^{\prime}(2)}{\zeta(2)}\right] .
\end{aligned}
$$

The dependence on $\mathrm{m}^{2}$ disappeared. Instead the infrared divergence is regulated by the intermediate momentum cut-off $q^{*}$.

The real photon emission rate is finally obtained by adding the soft, (17), and the hard, (24), contribution,

$E \frac{\mathrm{d} W^{\gamma}}{\mathrm{d}^{3} k} \simeq \frac{e_{q}^{2} \alpha \alpha_{\mathrm{s}}}{2 \pi^{2}} T^{2} \mathrm{e}^{-E / T} \ln \left(\frac{c}{\alpha_{\mathrm{s}}} \frac{E}{T}\right)$,

with the constant $c$ under the logarithm given by

$$
c \simeq \frac{3}{2 \pi} \exp \left[\frac{1}{2}+\frac{\ln 2}{3}-\gamma+\frac{\zeta^{\prime}(2)}{\zeta(2)}-0.31\right] \simeq 0.23 \text {. }
$$

Indeed the emission rate is seen to be independent of the otherwise arbitrary cut-off $q^{*}$. It contains, however, a logarithmic dependence on the strong coupling constant.

\section{Discussion}

We are now able to compare this result with previous estimates for the thermal differential photon rates. In par- ticular in the analysis by Neubert [7] no screening mechanism is introduced. Instead the regulator mass $m$ in (23) is interpreted as the bare quark mass $m_{q}$. In this case the corresponding rate is approximated by

$E \frac{\mathrm{d} W^{\gamma}}{\mathrm{d}^{3} k} \simeq \frac{e_{q}^{2} \alpha \alpha_{\mathrm{s}}}{2 \pi^{2}} T^{2} \mathrm{e}^{-E / T} \ln \left[c_{N}\left(\frac{T^{2}}{m_{q}^{2}}\right) \frac{E}{T}\right]$,

with the constant

$c_{N}=4 \exp \left[-\frac{1}{2}-\gamma+\frac{\ln 2}{3}+\frac{\zeta^{\prime}(2)}{\zeta(2)}\right] \simeq 0.97$,

which is larger than the one of (25). This is especially true when a small quark mass of e.g. $m_{q}=5 \mathrm{MeV}$ is used in applications with temperatures $T>100 \mathrm{MeV}$.

Kajantie and Ruuskanen [2] estimated the shielding effect in the quark-gluon plasma by giving the exchanged quark in the processes of (18) and (19) the thermal mass $m_{f}$ instead of $m_{q}$. This is achieved when the complete fermion propagator (cf. (6)) is approximated by a particle excitation only, i.e. by $\mathrm{i} q /\left(q^{2}-m_{f}^{2}+\mathrm{i} \varepsilon\right)$. Then the rate is of the form (25) with the constant $c$ replaced by $3 c_{N} / 2 \pi \simeq 0.46$, which is bigger than the complete result in (26) by a factor of 2.

Finally one may compare with the popular approximation $[5,7]$ using thermal Boltzmann-Maxwell distributions in the initial state instead of $n_{B}$ and $n_{F}$, respectively. Together with the replacement $m \rightarrow m_{f}$ this leads to

$\left.E \frac{\mathrm{d} W^{\gamma}}{\mathrm{d}^{3} k}\right|_{\mathrm{MB}} \simeq \frac{4 e_{q}^{2} \alpha \alpha_{\mathrm{s}}}{\pi^{4}} T^{2} \mathrm{e}^{-E / T} \ln \left(\frac{c_{M B}}{\alpha_{\mathrm{s}}} \frac{E}{T}\right)$,

with

$c_{M B}=\frac{6}{\pi} \exp \left(-\frac{1}{4}-\gamma\right) \simeq 0.84$,

an overestimate when compared with the constant $c$. The overall normalization factor differs by $8 / \pi^{2} \simeq 0.81$ from (25).

The comparison between our result for the thermal photon production rate (25) and the previous estimates is summarized in Fig. 3 in form of ratios: the dot-dashed curve describes the ratio of (27) to (25) as a function of the photon energy at $T=200 \mathrm{MeV}$ and for $m_{q}=5 \mathrm{MeV}$; the dashed curve shows the corresponding ratio identifying $m_{q}$ in (27) by the thermal mass $m_{f}$ given by (9); the solid line is for the ratio of (29) to (25). In summary one deduces from this figure that the screening mechanism due to Landau damping reduces significantly the production rate of thermal photons in particular at low photon energies.

In Fig. 4 the transverse momentum spectra of thermal photons produced in central heavy-ion collisions $A+B \rightarrow \gamma+X$ are plotted. The curves are obtained from the emission rate of (25) after integrating over the thermal evolution using the model by Bjørken [27]. The necessary integrations are performed for the realistic conditions of large transverse momenta $p_{T}$ compared to the thermalization temperature $T_{i}$, which itself is assumed to be large when compared to the critical temperature $T_{c}$ for the transition to the quark-gluon phase.

In the kinematical region for $p_{T}>2 \mathrm{GeV} / c$ and vanishing photon rapidity $y=0$ the spectrum is well approxim- 


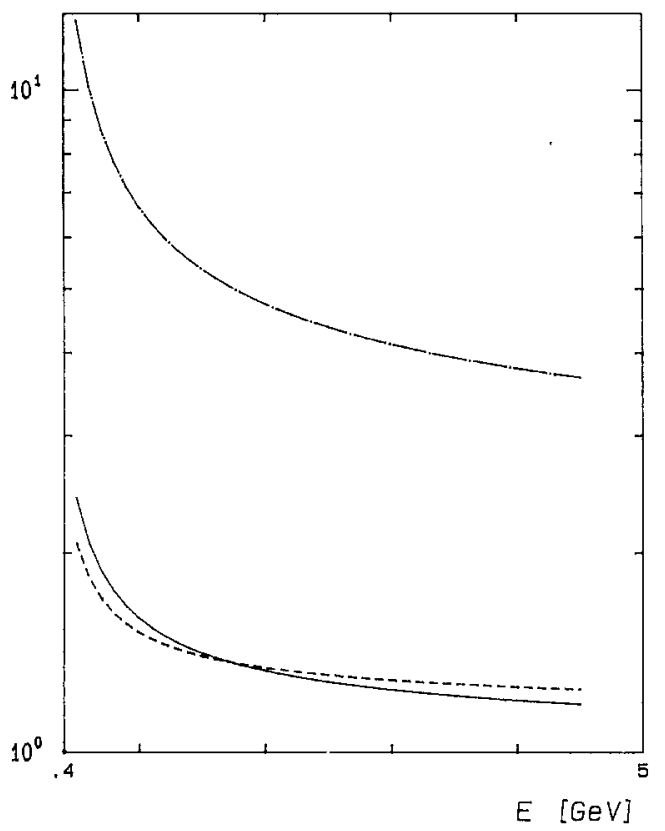

Fig. 3. Ratios of thermal photon production rates with respect to (25) as a function of the photon energy at $T=200 \mathrm{MeV}$. The dotdashed curve describes the ratio of (27) to (25) for $m_{q}=5 \mathrm{MeV}$; the dashed one shows the corresponding ratio identifying $m_{q}$ by the thermal mass; the solid line is for the ratio of (29) to (25)

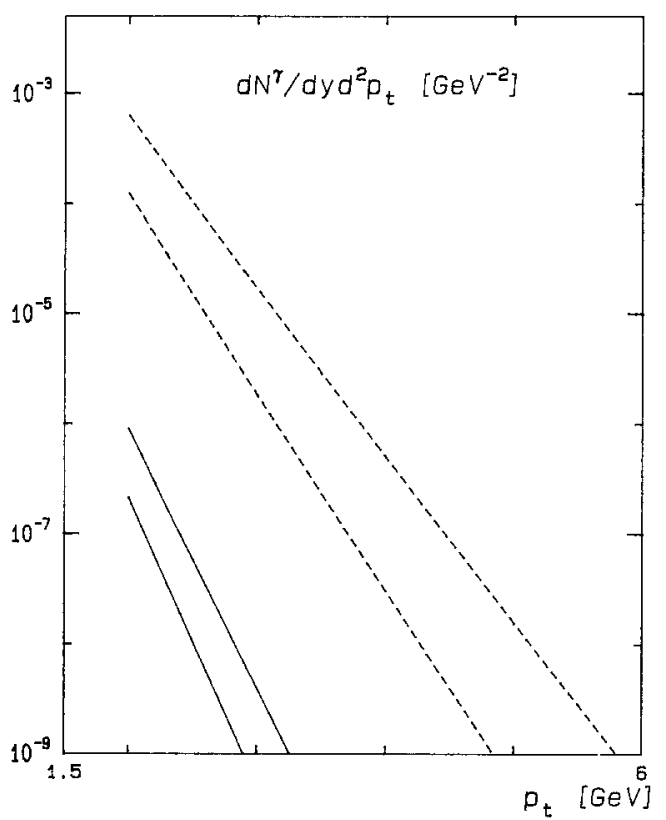

Fig. 4. Transverse momentum spectra for thermal photons for central heavy ion-collisions from (31). Solid curves correspond to $\mathrm{O}+$ Au collisions at CERN SPS energy calculated with: $\mathrm{d} N / \mathrm{d} y \simeq 87$, $T_{i}=180 \mathrm{MeV}$ (lower curve) and $\mathrm{d} N / \mathrm{d} y \simeq 119, T_{i}=200 \mathrm{MeV}$ (upper curve). Dashed curves are for $\mathrm{Pb}+\mathrm{Pb}$ collisions at LHC energy with: $\mathrm{d} N / \mathrm{d} y \simeq 1500, T_{i}=263 \mathrm{MeV}$ (lower curve) and $\mathrm{d} N / \mathrm{d} y \simeq 2450$, $T_{i}=310 \mathrm{MeV}$ (upper curve) ated by

$$
\begin{aligned}
\frac{\mathrm{d} N^{\gamma}}{\mathrm{d} y \mathrm{~d}^{2} p_{T}} \simeq & \frac{5 \sqrt{2 \pi} \alpha \alpha_{\mathrm{s}}}{6 \pi^{2}}\left(T_{i}^{3} \tau_{i}\right)^{2} \pi R_{A}^{2} \frac{\left[p_{T} / T_{i}\right]^{2.5}}{p_{T}^{4}} \\
& \cdot \ln \left(\frac{c}{\alpha_{\mathrm{s}}} \frac{p_{T}}{T_{i}}\right) \mathrm{e}^{-p_{T} / T_{i}},
\end{aligned}
$$

taking into account two quark flavours. According to $[5,27]$ the initial temperature $T_{i}$ at a thermalization time $\tau_{i}=1 \mathrm{fm}$ may be related to the central hadron multiplicity $\mathrm{d} N / \mathrm{d} y$ by

$\left[\frac{T_{i}}{100 \mathrm{MeV}}\right]^{3} \simeq 0.4 A^{-2 / 3} \frac{\mathrm{d} N}{\mathrm{~d} y}$

The typical shapes of the transverse momentum spectra of photons for $\mathrm{O}+\mathrm{Au}$ collisions at CERN SPS [1] and for $\mathrm{Pb}+\mathrm{Pb}$ collisions at LHC energies [8] are shown in Fig. 4 . The strong coupling constant is fixed by $\alpha_{s}=0.3$.

\section{Concluding remarks}

In this note an explicit calculation demonstrates that mass singularities due to the exchange of massless quarks are shielded in a quark-gluon plasma at high temperatures. This generalizes results recently derived in the case of exchanged massless gauge bosons. As a consequence of Landau damping and resummation this screening mechanism allows for infrared stable predictions for the emission rate of energetic real photons from hot quark-gluon matter. The constant under the logarithm $\ln \left(\frac{1}{\alpha_{s}} \frac{E}{T}\right)$ (cf. (25)) is determined. The presence of this logarithmic dependence on the coupling constant in the final expression reflects the resummed perturbative terms arising from the effective quark propagator. It also indicates that for soft external photons with energies or transverse momenta of $\mathcal{O}\left(\sqrt{\alpha_{\mathrm{s}}} T\right)$ or even of $\mathcal{O}\left(\alpha_{\mathrm{s}} T\right)$ additional screening processes [28]-similar to the dilepton case [26]-have to be included into the theoretical description.

Acknowledgements. We thank R. Santo for useful remarks on the experiment by the WA80 Collaboration. One of us (K. R.) is indebted to P.V. Ruuskanen and to D. Seibert for discussions and for providing information about the related work of [18] prior to publication. Discussions with $\mathrm{H}$. Satz are kindly acknowledged.

\section{References}

1. R. Albrecht et al., WA80 Collab: Z. Phys. C-Particles and Fields 51 (1991) 1

2. K. Kajantie, H.I. Miettinen: Z. Phys. C-Particles and Fields 9 (1981) 341; and earlier references quoted therein

3. K. Kajantie, P.V. Ruuskanen: Phys. Lett. B121 (1983) 352

4. B. Sinha: Phys. Lett. B128 (1983) 91

5. R.C. Hwa, K. Kajantie: Phys. Rev. D32 (1985) 1109

6. G. Staadt, W. Greiner, J. Rafelski: Phys. Rev. D33 (1986) 66

7. M. Neubert: Z. Phys. C-Particles and Fields 42 (1989) 231

8. P.V. Ruuskanen: Proceedings of ECFA Workshop on Large Hadron Colliders, Aachen 1990, Vol. II, p. 1164, G. Jarlskog, D. Rein (eds.), CERN 90-10 (1990); H. Satz, ibid. Vol. I, p. 188 
9. R. Baier, B. Pire, D. Schiff: Phys. Rev. D38 (1988) 2814; T. Altherr, P. Aurenche, T. Becherrawy: Nucl. Phys. B315 (1989) 436; T. Altherr, P. Aurenche: Z. Phys. C-Particles and Fields 45 (1989) 99; T. Altherr, T. Becherrawy: Nucl. Phys. B330 (1990) 174; J. Cleymans, I. Dadic: Z. Phys. C-Particles and Fields 42 (1989) 133; T. Grandou, M. Le Bellac, J.-L. Meunier: Z. Phys. C-Particles and Fields 43 (1989) 575

10. A. Niégawa, K. Takashiba: Preprint Bielefeld University BI-TP 91/12 (1991)

11. R.D. Pisarski: Nucl. Phys. B309 (1988) 476; Phys. Rev. Lett. 63 (1989) 1129

12. R.D. Pisarski: Nucl. Phys. A525 (1991) 175c

13. E. Braaten, R.D. Pisarski: Phys. Rev. Lett. 64 (1990) 1338; Nucl. Phys. B337 (1990) 569; Nucl. Phys. B339 (1990) 310

14. J. Frenkel, J.C. Taylor: Nucl. Phys. B334 (1990) 199

15. For a review: N.P. Landsman, Ch.G. van Weert: Phys. Rep. 145 (1987) 141; J.I. Kapusta: Finite-temperature field theory. Cambridge: Cambridge University Press 1989; M. Le Bellac: Lectures given at the XXXth Schladming Winter School, Schladming, 1991, preprint University of Nice INLN 1991/6

16. G. Baym, H. Monien, C.J. Pethick, D.G. Ravenhall: Phys. Rev. Lett. 64 (1990) 1867; G. Baym, H. Monien, C.J. Pethick: Proceedings of Int. Workshop on Gross Properties of Nuclei and Nuclear Excitations XVI, Hirschegg, Austria, 1988. H. Feldmeier; C.J. Pethick, G. Baym, H. Monien, (eds.), Nucl. Phys. A498 (1989) 313c
17. E. Braaten, T.C. Yuan: Phys. Rev. Lett. 66 (1991) 2183; E. Braaten, M. Thoma: Phys. Rev. D44 (1991) 1298

18. An independent treatment is given by: J.I. Kapusta, P. Lichard, D. Seibert: High energy photons from quark-gluon plasma versus hot hadronic gas, preprint University of Minnesota, 1991; and private communication

19. H.A. Weldon: Phys. Rev. D28 (1983) 2007

20. R.L. Kobes, G.W. Semenoff: Nucl. Phys. B260 (1985) 714; B272 (1986) 329

21. A. Niégawa: Phys. Lett. B247 (1990) 351; N. Ashida, H. Nakkagawa, H. Yokota, A. Niégawa: Preprint Osaka City University OCU-137 and OCU-138 (1991)

22. T. Altherr: Z. Phys. C-Particles and Fields 47 (1990) 559; Ann. Phys. (N.Y.) 207 (1991) 374

23. V.V. Klimov: Sov. J. Nucl. Phys. 33 (1981) 934; O.K. Kalashnikov: Fortschr. Phys. 32 (1984) 525

24. H.A. Weldon: Phys. Rev. D26 (1982) 2789; Physica A158 (1989) 169; Phys. Rev. D40 (1989) 2410

25. R.D. Pisarski: Physica A158 (1989) 146; Fermilab preprint Pub88/113-T (unpublished)

26. E. Braaten, R.D. Pisarski, T.C. Yuan: Phys. Rev. Lett. 64 (1990) 2242

27. J.D. Bjørken: Phys. Rev. D27 (1983) 140

28. E. Braaten: Nucl. Phys. B (Proc. Suppl.) 23B (1991) 351 